\title{
Visceral Leishmaniasis Turning into Chronic Liver Disease
}

\author{
SHEGUFTA MISHKET MUKERRAMA,${ }^{1}$ AHMEDUL KABIR, ${ }^{2}$ SUDIP RANJAN DEB, ${ }^{3}$ AVISHEK SARKER DHRUBA, ${ }^{1}$ \\ PRATYAY HASAN, ${ }^{1}$ ANWAR HOSSAIN,${ }^{4}$ MAHBUB HOSSAIN ${ }^{5}$
}

\begin{abstract}
Kala-azar and chronic liver disease are conditions frequently seen in our country. In some instances, when features of both diseases co-exist, it may pose as a diagnostic dilemma. We intend to report a case here, where presence of such features led to much confusion.
\end{abstract}

\section{Introduction}

Kala-azar is a very common disease in Bangladesh. An individual in an endemic area who has fever for more than 3 weeks, splenomegaly \& rK-39 test is positive should be diagnosed as a case of kala-azar. Kala-azar is a disease caused by parasites of genus Leishmania, which are transmitted by Phlebotomine sandflies. The infection may be anthroponotic or zoonotic. On the other hand, cirrhosis of liver is defined histopathologically. It includes decrease in hepatocellular mass, development of fibrosis to the point that there is architectural destruction with formation of regenerative nodule. The induction of fibrosis occurs with activation of stellate cell, resulting in increased formation of collagen and other components of intracellualar matrix. However, the association of chronic liver disease with visceral leishmaniasis is not well established. We report this case because we have found both visceral leishmaniasis(in liver) $\&$ chronic liver disease in our patient. The presenceofboth etiologies has been proved by liver biopsy. If the visceral leishmaniasis is the cause of chronic liver disease, this may be the first case report in the world of a patient of chronic liver disease due to visceral leishmaniasis.

\section{Case Report}

A32 years old male, by profession a school teacher, hailing from Faridpur, was presented to us on 15.11.13 with the complaints of vomiting for 4 episodes \& history of unconsciousness for 2 days. According to the statement of

1. Medical Officer, Department of Medicine, Dhaka Medical College \& Hospital, Dhaka

2. Associate Professor, Department of Medicine, Dhaka Medical College \& Hospital, Dhaka

3. Assistant Professor Department of Medicine, Dhaka Medical College \& Hospital, Dhaka.

4. Assistant Registrar, Department of Medicine, Dhaka Medical College \& Hospital, Dhaka.

5. Indoor Medical Officer, Department of Medicine, Dhaka Medical College \& Hospital, Dhaka.

Correspondence: Dr. Shegufta Mishket Mukerrama, smm_ayontika@yahoo.com. Contactno-01726842566 his relatives, he was quite well before February 2012. Then he developed fever which was high grade, associated with chills and rigor, anorexia \& weight loss. He consulted several physicians and took several courses of antibiotics and antipyretic but no improvement occurred. They took him to Kolkata, India in August, 2012. On 23rd November, 2012 splenectomy was done due to autoimmune haemolytic anaemia with pancytopenia (as per noted in his discharge note from India). Before operation, he took several courses of steroid but no pre-operative vaccination was given. Biopsy of resected spleen was done but the report was not given to the patient. No post-operative vaccination was advised. He was quite well after the operation upto this time when he was treated as a case of Meningoencephalitis in a local hospital in Faridpur before his admission in our hospital. He is nondiabetic, non-asthmatic \& has no history of Hypertension or contact with TB patient. Patient gave no history of yellow discoloration of sclera, urine or stool, abdominal distension, haematemesis or melaena, chest pain, respiratory distress or haemoptysis.

On admission, he was ill-looking with average body build $\&$ nutritional status, unconscious, anaemic, there was no

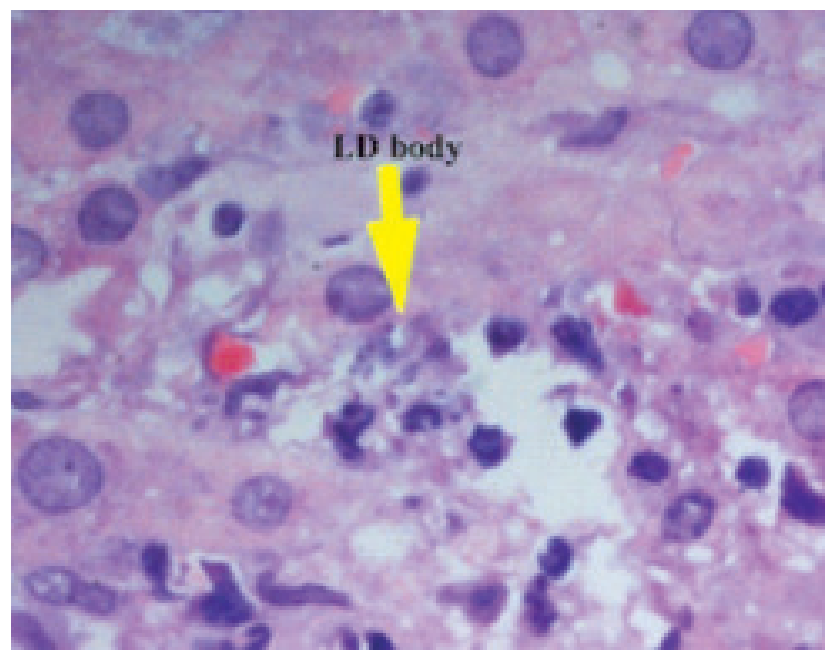

Fig. -1: Photomicrograph (H\&E staining, x1200) showing single L D body 
jaundice, cyanosis or oedema, temperature was raised. GCS of the patient was 10. Plantar response was equivocal on both sides. Bilateral gynaecomastia \& palmar erythema on both hands were found, two spider naevi were found in the trunk. There was a scar mark of left subcostal incision. His blood pressure was 100/60 $\mathrm{mm} \mathrm{Hg}$, pulse was 72beats/min, temperature was slightly raised. Regarding systemic examination, there was no engorged vein, hair distribution was normal. Liver was moderately enlarged in size, about $6 \mathrm{~cm}$ from the right costal margin along the mid clavicular line, surface was smooth with regular margin, firm in consistency, non-tender, moves with respiration. Upper border of liver dullness was in right $5^{\text {th }}$ intercostal space along the right mid-clavicular line \& hepatic bruit was absent. Bowel sound was present. There was no ascites. Other systemic examinations revealed no abnormality.

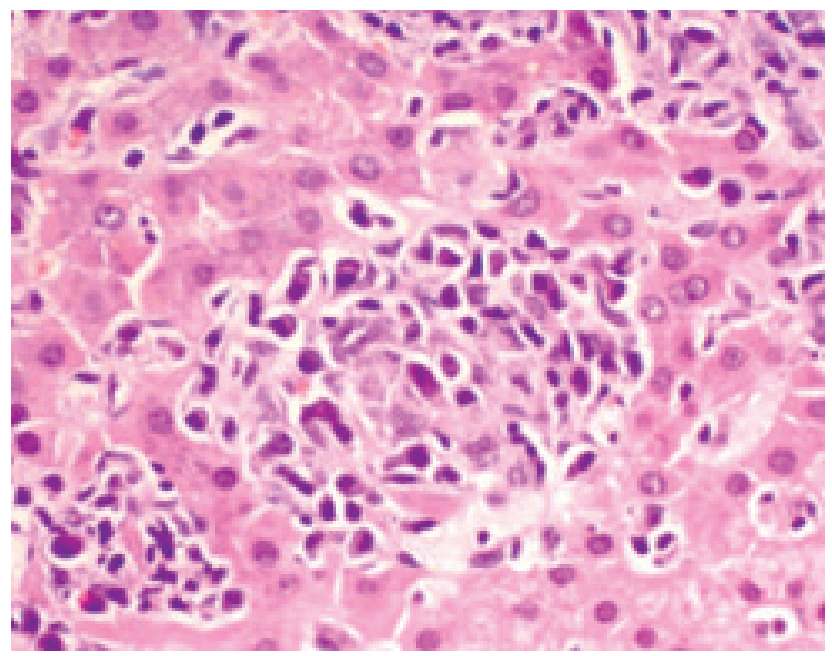

Fig.-2: Photomicrograph showing nodularity in the liver

We investigated the patient\& found that his haemoglobin level was $9.89 \mathrm{gm} / \mathrm{dl}$, ESR $82 \mathrm{~mm}$ in $1^{\mathrm{st}} \mathrm{hr}$, total WBC count 13,500/cumm,differential count: Neutrophils-75\%, Lymphocytes- $21 \%$, Platelet count-1,40000/cu mm, RBCs were normocytic \& normochromic. Liver function test showed SGPT: 101U/L, SGOT:82U/L, Total protein: 114gm/ L, Serum Albumin: 33gm/L, Serum Globulin:81 gm/1, A:G ratio was $4: 1$ (altered). Prothombin time:12.2 seconds. Serum Ammonia: 44 micromol/L(Normal:9-30 micromol/ L).S.LDH: 295 U/L. Serum Creatinine, Serum electrolytes, Blood Glucose\& Serum Vitamin B12 \&Folatewerewithin normal limit. Chest X-ray P/A view \& ECG: Normal. Upper GI Endoscopy \& CT scan of brain was normal. USG ofW/A showed hepatomegaly with sludge in gall bladder.Anti Smooth muscle Antibody\&Anti NuclearAntibody(ANA) were Negative.Serum CPK:24U/L, HBsAg, Anti HCV Antibody, Anti HBc(Total), HIV 1 \& 2were Negative.

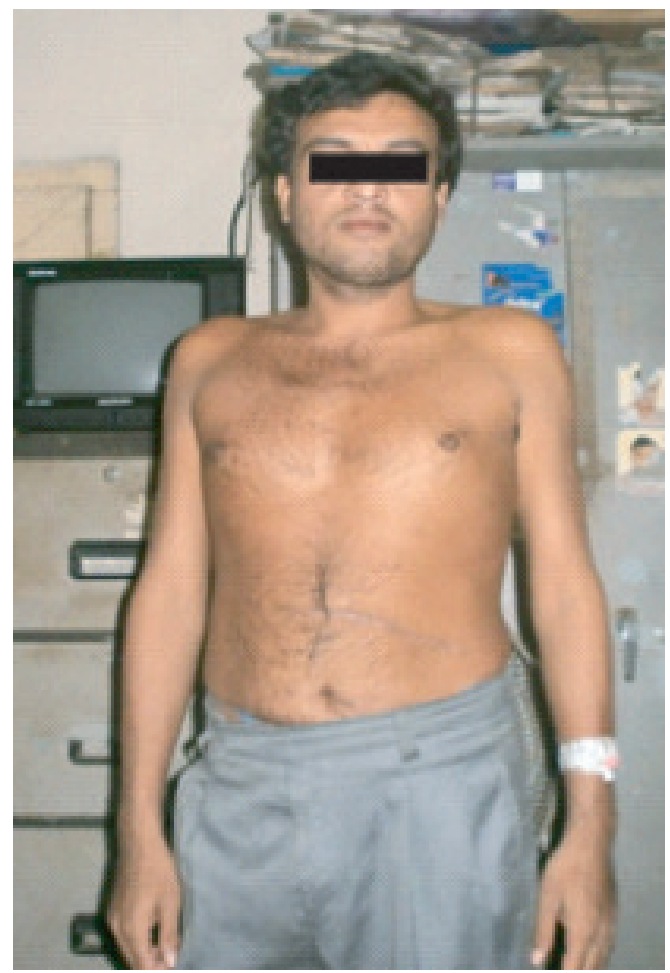

Fig.-3: Appearance of the Patient

Fibroscan of Liver : Liver stiffness is $6 \mathrm{Kpa}$ which is normal.(N.B.-IN $15 \%$ case although fibrosis is present report may be normal,Infibroscan, advanced fibrosis may be underestimated \& patient with macronodular cirrhosis may be classified as non-cirrhotic.)ICT for KALAAZAR(24.11.13): Positive (from BSMMU) which was again repeated on $26.11 .13 \&$ found to be Positive (fromIEDCR).

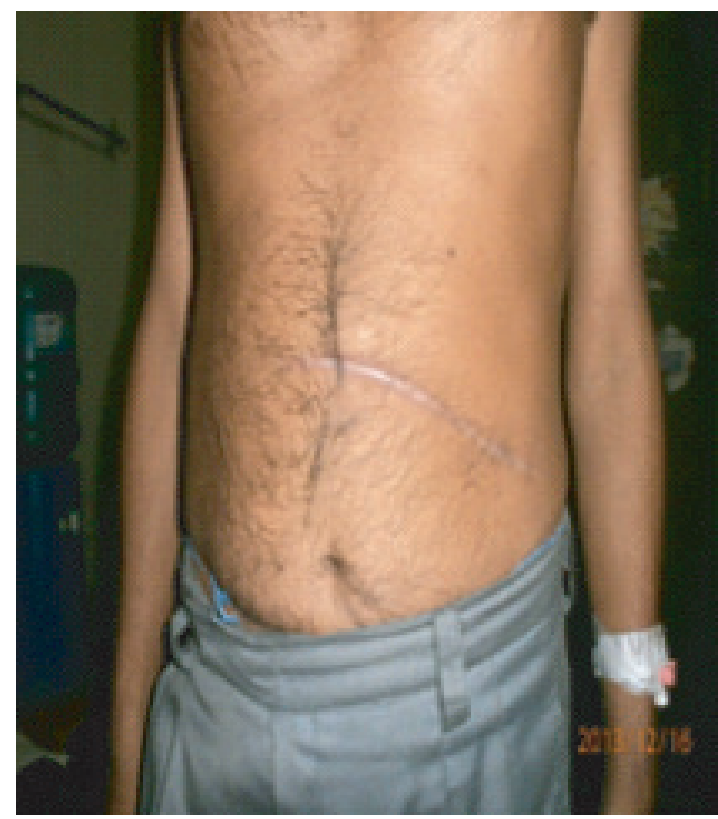

Fig.-4: Splenectomy scar 


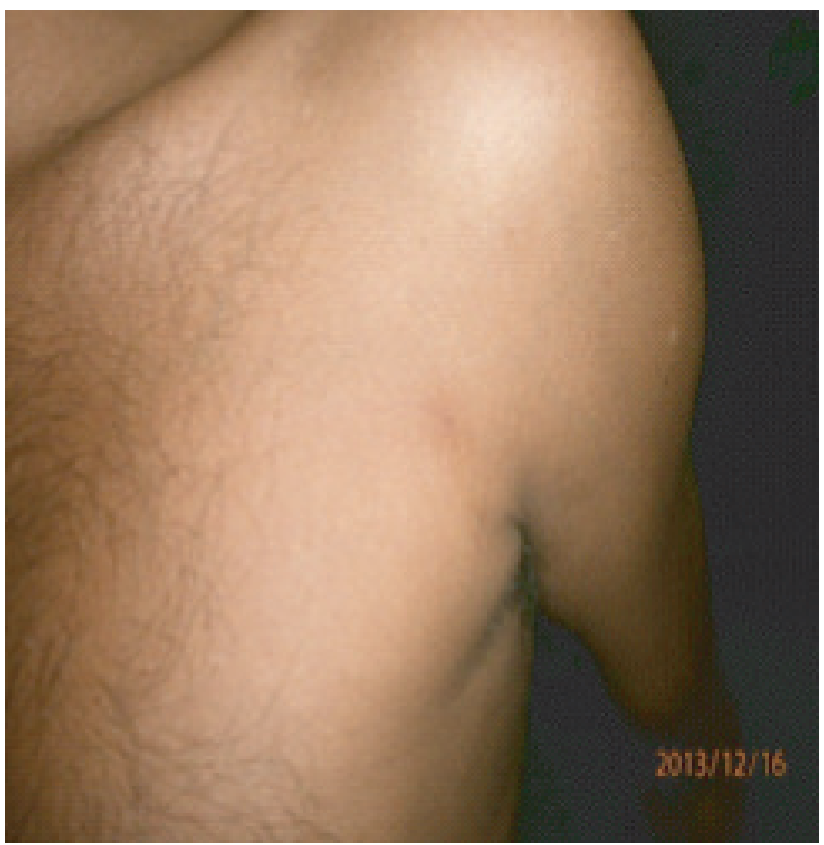

Fig.-5: Spider angioma on the trunk

Bone marrow examination revealed (2.12.13): Reactive marrow with Plasma cell: 8-10\%.Bonemarrow slide was again reviewed on $4.12 .13 \&$ comment was:No malignant cell or granuloma, no LD body. Smears revealed normal haematopoietic cells. Haemoglobin with blood film done on 23.11.13showed: $\mathrm{Hb}-13.7 \mathrm{gm} / \mathrm{dl}, \mathrm{MCV}-84.9 \mathrm{fl}$, Reticulocyte count-2.64\%,PBF: RBC is normocytic and normochromic, WBC mature with increased number, Comment is leukocytosis.Investigations done previously in India one year back showed:Direct Coomb's Test :Positive(31.8.12) Upper GI Endoscopy: AntralGastritis. CBC: Hb-8gm/dl, Total count of WBC-9200/cumm, Neutrophil:40\%, Lymphocyte: 54\%(29.8.2012). Another

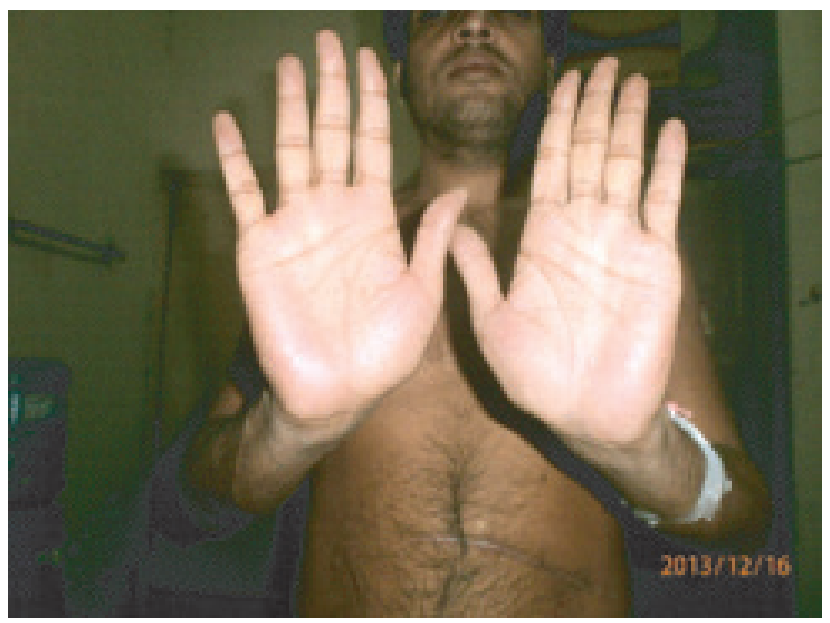

Fig.-6: Palmar erythema in the patient
CBC:Hb-8.2gm/dl,Platelet-1,8,0000/cumm (20.9.12). Serum ferritin: 436ng/ml(19.9.12), Reticulocyte count: 5.19\%(20.9.12) \&2.38\%(30.8.12). Total Billirubin:0.5 mg/ dl,direct: $0.2 \mathrm{mg} / \mathrm{dl}$, Indirect: $0.3 \mathrm{mg} / \mathrm{dl}$. Trephine biopsy showed megaloblastic change (9.10.12) Bone marrow report: Suggestive of megaloblastic anaemia(9.10.12).

For final confirmation,we did liver biopsyon $9^{\text {th }}$ December 2013. The result of liver biopsy showed-periportal fibrosis and moderate infiltrate of chronic inflammatory cells. The histological Activity Index was 10/18 (Periportal +/- bridging necrosis $=4$, Intralobular degeneration and focal necrosis $=3$, Portal inflammation $=3$, Fibrosis $=1$ ). The knodell score was 11/22. Further section revealed a few LD bodies within histiocytes in the portal areas. Kuppfer cells were prominent.

After confirmation of chronic liver disease \& visceral leishmaniasis, patient was treated with single dose of Liposomal amphotericin B (10 mg per kg of body weight). Gut sterilizer \& lactulose was also started. The patient responded well. Patient was vaccinated against all encapsulated organisms before discharge. Patient was also advised to take life long penicillin.

\section{Discussion}

Visceral Leishmaniasis or Kala-azar is a deadly disease putting 350 million people from 88 countries at risk with an annual new case burden of 1.5 to 2 million and the number is rising. ${ }^{1}$ Of all the cases, $90 \%$ occur in India, Bangladesh, Nepal, Sudan and Brazil. A total of 3.50 million people are at risk. ${ }^{2}$ In our country, chronic liver disease is fairly common $\&$ often seen whereas, Visceral Leishmaniasis also puts significant disease burden to our community. Previous epidemiological studies shows that, 34 out of 64 districts of Bangladesh reported Kala-azar cases but $90 \%$ of them are from 10 districts. ${ }^{3}$ The worst hit area in Bangladesh is Mymensingh followed by Pabna, Tangail, Jamalpur, Sirajganj, Gazipur, Natore, Naogaon, Manikganj, Rajshahi and Naawabgaj. Our patient is from Faridpur area, which is just adjacent to the above mentioned Kala-azar prone areas. ${ }^{3}$ Kala-azar is often confused with chronic liver disease in clinical practice. Many recent instances can be given. In a recent Bangladeshi study, it is found that initial diagnosis of chronic liver disease was made in $36 \%$ of the patient with visceral leishmaniasis \& same study also mentions that $9 \%$ of Kala-azar patient was initially thought to have congenital hemolytic anaemia. ${ }^{4}$ In India, multiple cases are reported where visceral leishmaniasis was mistaken for chronic liver disease $\&$ treatment was delayed which ultimately proved to be fatal. ${ }^{5,6}$ In these cases, correct diagnosis was delayed mainly due to absence of features characteristic of visceral leishmaniasis, such as fever. Also many rare events were 
superimposed, such as, severe acute hepatic involvement or acute liver failure. ${ }^{7}$ It is noteworthy that our case also had significantly raised gamma globulin level which is similar to the case mentioned before. ${ }^{7}$ Our patient had gone through splenectomy operation in the year 2012 in India, due to suspicion of hypersplenism, which added to the confusion of the true diagnosis of the patient. We found spider angioma, palmar erythema, gynaecomastia; all of them favour diagnosis of CLD.And initial presentation during admission was unconsciousness which was then thought to be possibly caused by metabolic derangement as a result of chronic liver disease. It was also confused with overwhelming postsplenectomy sepsis. After exclusion of all the possible causes of chronic liver disease \& on the background of endemicity of the patient's residential area \& nature of his household,previous history of pancytopenia, and significantly raised serum globulin level, we had to consider Kala-azar also. Kala-azar often involves the liver due to effect of LD body itself or immunological response against it leading to altered biochemistry of liver. ${ }^{4} \mathrm{We}$ investigated thoroughly pursuing both lines of diagnoses. Upper GI endoscopy \& fibroscan of liver were normal. USG of abdomen showed hepatomegaly with normal echo-texture of liver. So we could not find any evidence in favor of chronic liver disease. Moreover, we could not take splenic aspirate due to previous splenectomy \& bone marrow examination revealed only elevated plasma cell count. In spite of all the negative features, under strong clinical suspicion, we sent blood for rK39 test \& liver biopsy was taken. rK39 test has sensitivity of $100 \%$ and specificity of $95 \% .{ }^{8}, 9$ Liver biopsy is the gold standard \& ultimate diagnostic tool for the detection of liver pathology in spite of normal fibro scan of liver. ${ }^{10}$ Liver biopsy and rk39 both showed result in favour of cirrhosis and Kala-azar. So, we ultimately concluded that our patient had both Kala-azar and chronic liver disease. And in absence of any specific etiology for chronic liver disease, we came to believe that, these two conditions may have cause-effect relationship.

\section{Conclusion}

In some instances, we find it difficult to distinguish between chronic liver disease and Kala-azar. When presented with such difficulty, under strong clinical suspicion, we need to pursue both lines of diagnoses. Specifically in this case, where both chronic liver disease and Kala-azar are confirmed by gold standard of investigation, it comes to mind that Kalaazar may have caused chronic liver disease in this patient. We recommend further study on this subject matter.

\section{Conflict of Interest : None}

\section{References}

1. Desjeux P. Global control and Leishmania HIV co-infection. Clin Dermatol. 1999;17(3):317-25.

2. Ahsan M HAMN, Mohammed FR, Chowdhury FR, Alam. Treatment of Kala-azar: past, present \& future. J Medicine. 2008; 9:90-95.

3. Bern C, Chowdhury R. The epidemiology of visceral leishmaniasis in Bangladesh: prospects for improved control. Indian J Med res . 2006;123(3):275-88.

4. Rashid A, Mamun A, Rasul C, Afrafuzzaman M, Hossain M, Rahman M. Jaundice in Pediatric Visceral Leishmaniasis (Kala-azar) Patients. J Medicine. 2008;8(1).

5. Mohan A, Vishnuvardhan Reddy E, SamantarayJC, Sharma SK. A rare presentation of visceral leishmaniasis without fever or splenomegaly in an elderly person.Eur J Int Med. 2007;18(2):158-60.

6. Prakash A, Singh NP, Sridhara G, Malhotra V, Makhija A, Garg D, et al. Visceral leishmaniasis masquerading as chronic liver disease. J AssocPhys India. 2006;54:893-4.

7. Sagnelli C, Di Martino F, Coppola N, Crisci A, Sagnelli E. Acute liver failure: a rare clinical presentation of visceral leishmaniasis. New Microbiologica. 2012;35(1):93-5.

8. Bern C, JhaSN, Joshi AB, Thakur GD, Bista MB. Use of the recombinant $\mathrm{K} 39$ dipstick test and the direct agglutination test in a setting endemic for visceral leishmaniasis in Nepal. Am J Trop Med Hyg. 2000;63(3-4):153-7.

9. Sundar S, Reed SG, Singh VP, Kumar PC, Murray HW. Rapid accurate field diagnosis of Indian visceral leishmaniasis.Lancet. 1998;351(9102):563-5.

10. Nudo CG, Jeffers LJ, Bejarano PA, Servin-Abad LA, Leibovici Z, De Medina M, et al. Correlation of Laparoscopic Liver Biopsy to Elasticity Measurements (FibroScan) in Patients With Chronic Liver Disease. Gastroenterol Hepatol. 2008;4(12):862-70. 